\title{
An Accurate Model of Beam Ion Instability with Nonlinear Space Charge, Realistic Beam Optics and Multiple Gas Species Vacuum
}

\author{
L. Wang", M. Pivi, T. O. Raubenheimer and J. Safranek \\ SLAC National Accelerator Laboratory, Menlo Park, CA, USA
}

\section{Abstract}

The previous analyses of beam ion instability have been performed for single gas species only. However, there are multiple gas species in the vacuum chambers of an accelerator. The superposition rule doesn't apply in general and it overestimates the instability. Therefore, it is important to use multiple gas species model. On the other hand, the variation of beam size along the accelerator ring or linac provides Landau damping to the instability. In previous studies, the effect of beam optics has been represented by a frequency spread and a quality factor. In practice, it could be difficult to accurately estimate the frequency spread and quality factor for general beam optics because the variation of the beam size along the ring usually doesn't have simple distribution. This paper provides a more accurate method to analyze beam ion instability with arbitrary vacuum component and arbitrary beam optics where the variation of the beam size along the accelerator ring or linac can be arbitrary. Meanwhile, the nonlinear space charge effect is also included and the general beam filling pattern can be easily modeled. Our analyses agree well with expensive simulations.

\section{PACS numbers: 29.27.Bd, 29.20.db}

\section{INTRODUCTION}

In accelerators with negatively charged beams, for instance electron accelerator, ions generated from the residual gas molecules due to beam gas ionization can be trapped by the beam. The trapped ions interact resonantly with the beam and cause a beam-ion coherent instability, tune shift and emittance blow-up. This beam-ion instability can occur in both rings and linacs. The beam emittance of future accelerators will be at the ultra small, picometer level where strong beam ion instability can occur. The analysis of beam ion instability has been done for single bunch train [1-6] and multi-bunch train beam filling patterns [7]. A number of important factors need to be properly modeled in order to give accurate analysis. These key factors include nonlinear space charge force, realistic multiple gas species and beam optics effect. All the effects contribute to make the instability weaker.

In all previous analyses, a single gas species model has been used. In the real vacuum chamber, there are multiple gas species. A single gas-species model overestimates the beam ion instability. Multi-gas species can provide important

\# Email address: wanglf@slac.stanford.edu 
additional damping to the instability as shown later. Therefore, it is more accurate to use a multi-gas species vacuum model. This becomes essential for multi-bunch trains beam, since the efficiency of the beam filling pattern at reducing the ion density depends on both beam density and the mass of ions [7]. Therefore the reduction of the ion density by using multiple bunch train beam depends on the ion species. In fact gaps in the bunch train are more effective to reduce the density of lighter ions. Hence it is important to accurately model both the beam filling pattern and the vacuum simultaneously.

We discuss the beam ion instability with an electron beam in this paper. The electron bunch has Gaussian distribution and its space charge field has strong nonlinearity $[2,6,7]$. The strong nonlinear field of Gaussian distributed particles induces a large frequency spread, and hence causes a low $Q$ of the space charge wake function. In other words, the wake field decays quickly due to the nonlinear space charge force and it provides strong damping to the instability. Note that the growth rate of the instability is proportional to the $Q$ factor, or inversely proportional to the frequency spread $[6,7]$.

Ions are distributed along the whole accelerator beam chamber where the beam size usually varies significantly. Therefore, ions at different locations of the accelerator have different oscillation frequencies, so the ions couple to beam with different frequencies. This frequency spread induced by the beam optics provides Landau damping to the beam ion instability. The effect of beam optics was included in the previous analyses as a frequency spread [3] and a $Q$ factor [7]. However, it remains unanswered how to accurately calculate the frequency spread and $Q$ factor in practice because it is not straightforward to accurately determine them for a given arbitrary beam optics.

Therefore, it becomes crucial to simultaneously and accurately include the effects of multiple gas species, nonlinear space charge force and beam optics. This paper presents a more practical way of modeling all three factors together in a general case where the vacuum and the beam optics can be arbitrary. We apply the model to fill patterns with multiple short bunch trains followed by short gaps with empty bunches. Short gaps in the trains of electron bunches are a very effective mitigation of beam ion instability with high current and low emittance beams [7]. Therefore multiple bunch trains will be the choice of future high intensity electron accelerators. However our model is valid for any beam filling pattern, such as a single bunch train. The method used in this paper can be used for storage rings, linacs and extraction beam lines.

To be specific, we use SPEAR3 storage ring and the International Linear Collider (ILC) damping ring for our analyses. The SPEAR3 ring, like most light sources, has relatively small variation in betatron function along the ring. On the other hand, there is large variation in beam size in the ILC damping ring and Colliders, for instance PEPII, KEKB, SuperB and SuperKEKB. Table 1 lists the main parameters of SPEAR3 and the ILC DTC damping ring [8].

Table 1 Main Parameters of SPEAR3 and the ILC DTC damping Ring 


\begin{tabular}{|c|c|c|c|}
\hline Physics & Symbol/Unit & SPEAR3 & ILC DTC damping ring \\
\hline Beam Energy & $E_{0}[\mathrm{GeV}]$ & 3 & 5 \\
\hline Circumference & $C[\mathrm{~m}]$ & 234 & 3238.76 \\
\hline Horizontal Emittance & $\varepsilon_{\mathrm{x}}[\mathrm{nm}]$ & 10 & 0.637 \\
\hline Vertical Emittance & $\varepsilon_{\mathrm{y}}[\mathrm{pm}]$ & 14 & 2 \\
\hline Beam Current & $I[\mathrm{~mA}]$ & $200-500$ & $389 / 779$ \\
\hline Bunch Number & $M$ & 280 & $1312 / 2625$ \\
\hline Harmonic Number & $h$ & 372 & 7022 \\
\hline Bunch Spacing & $n s$ & 2.1 & $6.2 / 3.1$ \\
\hline RF Frequency & $f_{R F}[\mathrm{MHz}]$ & 476.315 & 650 \\
\hline Revolution Frequency & $f_{0}[\mathrm{MHz}]$ & 1.280 & 0.09 \\
\hline Tune & $v_{\mathrm{x}} / v_{\mathrm{y}} / v_{\mathrm{s}}$ & $14.1 / 6.18 / 0.01$ & $48.36 / 27.22 / 0.03$ \\
\hline Momentum Compaction Factor & $\alpha$ & $1.6 \times 10^{-3}$ & $3.36 \times 10^{-4}$ \\
\hline Energy Spread & $\sigma_{e}$ & $9.8 \times 10^{-4}$ & $1.0 \times 10^{-3}$ \\
\hline Bunch Length & $\sigma_{l}[\mathrm{~mm}]$ & 6 & 6 \\
\hline Radiation Damping Time & $\tau_{x} / \tau_{\mathrm{y}} / \tau_{z}[\mathrm{~ms}]$ & $4.0 / 5.3 / 3.2$ & $22 / 22 / 11$ \\
\hline Total Vacuum Pressure & $P[\mathrm{nTorr}]$ & $0.1 \sim 0.5$ & 0.5 \\
\hline
\end{tabular}

This paper is organized as follows. The wake function and impedance due to ion cloud with arbitrary beam optics, vacuum and beam filling pattern are presented in section II. In section III, a rigorous and direct method to analyze the instability with arbitrary beam optics and vacuum is described using both wake function and impedance. It clearly shows the importance of the multiple gas species model. The analyses of beam ion instability in the frequency domain in section IV gives a deeper understanding of the physics. The beam ion instability can be much better explained by the impedance than the wake function. There we also discuss the suppression of the beam ion instability by chromaticity and the way to estimate the frequency spread induced by the beam optics, which can be used in other analyses as used in [3,6,7]. Finally comparisons between simulations and analyses are given in section V.

\section{WAKE FUNCTION AND IMPEDANCE WITH ARBITARY BEAM OPTICS AND VACUUM}

The beam ion instability is two-stream beam instability. The ions generated by gas ionization are trapped by a negative charged beam (electron beam here). In the linear regime, the coupling force between the two-stream electronion beams can be described by a wake function. When $\omega_{i} S_{b} / c<<1$, the wake function of an ion-cloud with a constant beam size is given by [7]

$$
W_{y}(s)=\hat{W}_{y} e^{-\frac{\omega_{i} s}{2 Q_{0} c}} \sin \left(\frac{\omega_{i} s}{c}\right),
$$

with the amplitude of the wake as

$$
\hat{W}_{y}=N_{i}\left(\frac{r_{p} S_{b}}{A N_{e}}\right)^{1 / 2}\left[\frac{4}{3} \frac{1}{\sigma_{y}\left(\sigma_{y}+\sigma_{x}\right)}\right]^{3 / 2} .
$$


where $c$ is the speed of light, $r_{p}$ is the classical radius of proton, $A$ is the mass number of the ion, $N_{i}$ is the ion number, $N_{e}$ is electron bunch population, $S_{b}$ is bunch spacing and $\sigma_{x, y}$ is the transverse root mean square ( $r m s$ ) beam size of the electron bunches. $Q_{0}$ is the quality factor due to the nonlinear space charge field, which depends on the distribution of the particles. It is about 9 by simulation for a Gaussian distributed beam [7]. The nonlinearity varies with the vertical beam emittance when the horizontal emittance is fixed. For instance, the $Q_{0}$ value changes from 8.63 to 9.74 when the vertical emittance is increased from $1 \mathrm{~nm}$ to $8 \mathrm{~nm}$ in our simulation. The nonlinearity of the fields with other distributions can also be estimated numerically.

The wake function of an ion beam represents the space charge force between the ion-electron beams. But it is different from the traditional space charge wake and impedance. For the two-stream electron-ion beams, the leading electron bunch affects the following one via the ion beam. As a result, the wake function of the two-stream beams depends on the charge and distribution of the two beams. Note that the distribution of ion cloud is not exactly Gaussian distribution, but the electric field of the ion-cloud closely approximates that of a Gaussian distribution. The wake function given in Eqs.(1-2) is based on this approximation and it slightly underestimates the wake function of ion cloud [7]. For a flat beam as in most electron rings, the vertical wake function is larger than the horizontal one. Therefore, there is a stronger instability in the vertical plane. In the rest of this paper we will discuss about the instability in the vertical direction only and omit the subscript $y$ in some variables for simplicity. The coherent ion oscillation frequency is

$$
\omega_{i, y}=2 \pi f_{i, y} \approx c\left(\frac{4 N_{e} r_{p}}{3 A S_{b}\left(\sigma_{x}+\sigma_{y}\right) \sigma_{y}}\right)^{1 / 2}
$$

In general the beam size varies along the ring and the vacuum pressure can also be different from section to section along the ring. The total wake function from the ions can be calculated from the integral around the whole ring as

$$
W_{\text {ring }}(s)=\int_{0}^{c} \frac{4}{3} \frac{\omega_{i, y}(z)}{c} \frac{\lambda_{i}(z) S_{b}}{N_{e}} \frac{1}{\sigma_{y}(z)\left(\sigma_{y}(z)+\sigma_{x}(z)\right)} e^{-\frac{\omega_{i}(z) s}{2 Q_{0} c}} \sin \left(\frac{\omega_{i}(z) s}{c}\right) d z .
$$

Where $\lambda_{i}$ is the ion line density. The volume density of ion-cloud increases linearly along the bunch train and exponentially decays during the bunch train gap with a decay time of the order of ion oscillation period [7]. Therefore, the above equation can be used to calculate the wake function with arbitrary beam filling pattern. For instance, for a single bunch train consisting of $n_{b}$ bunches followed by a long gap [1-3], the ion line density at the end of bunch train is given by

$$
\lambda_{i}=\delta_{i} \frac{P}{k T} N_{e} \frac{L}{S_{b}}
$$


Here $P$ is the vacuum pressure, $\delta_{i}$ is the ionization cross-section, $L$ is the length of bunch train, $T$ is temperature and $k$ is

\section{Boltzmann constant.}

The total wake function with multiple gas species can be calculated from Eq.(4) by linearly adding the wake function of different ion species together. As a result, Eq.(4) gives the total wake function of ions along the ring with arbitrary beam optics and vacuum, which also includes the nonlinear space charge effect. It should be pointed out that it is essential to calculate the wake function using the integral in Eq. (4). The wake function can be very inaccurate if it is estimated by assuming a constant beam size along the ring as it is shown later.

The vacuum of different accelerators varies. Table 2 shows the measured vacuum components in the SPEAR3 chambers. They are the average values of four vacuum gauge data. Hydrogen is the dominant gas. We will use this vacuum component through this paper.

In most cases, it is clearer to look at the problem in frequency domain. The total impedance due to the ion cloud along the whole ring can be obtained by Fourier Transform of the wake function as

$$
Z_{\perp}(\omega)=i \int_{-\infty}^{\infty} \frac{d s}{c} W_{\text {ring }}(s) e^{i \omega s / c}
$$

The wake function and the impedance including all effects (nonlinear space charge force, beam optics and multiple gas species) in SPEAR3 for a total pressure of 0.5 nTorr is shown in Fig.1. The beam filling pattern consists of six bunch trains and a total beam current of $500 \mathrm{~mA}$. The ion density seen by different bunches varies when the beam is not uniformly distributed. The wake function is calculated using the average ion density seen by all bunches. To improve comprehension of the physics, the wake function and the impedance induced by different ion species are presented in order to compare the instability driven by different ion species. A quality factor $Q_{0}$ of 9 is used. The wake function of the $\mathrm{CO}_{2}$ ion species has the largest amplitude due to its large ionization cross-section and small diffusion rate during the train gap because of its large mass. $\mathrm{H}_{2}$ ion species has the second largest wake function due to the largest contribution to the vacuum pressure. The wake functions of each type ions in Fig. 1a still have resonance form. The wavelength of wake functions is longer than the bunch spacing. Therefore they can cause couple bunch instability. Similarly to the beam optics effect, the multi-gas species can also contribute to an additional spread in the ion frequency, as shown in

Fig. 1b. The overlapping of the frequencies of different types of ions species increases the overall ion frequency spread and therefore it further mitigates the instability. Note that the frequency of $\mathrm{H}_{2}$ ion is well separated from the frequencies of other ion species.

Table 2: Vacuum in the SPEAR3 beam chamber

\begin{tabular}{|l|l|l|l|}
\hline Gas Species & Mass Number & Cross-section (Mbarn) & Percent composition \\
\hline
\end{tabular}




\begin{tabular}{|c|c|c|c|}
\hline $\mathrm{H}_{2}$ & 2 & 0.35 & $48 \%$ \\
\hline $\mathrm{CH}_{4}$ & 16 & 2.1 & $5 \%$ \\
\hline $\mathrm{H}_{2} \mathrm{O}$ & 18 & 1.64 & $16 \%$ \\
\hline $\mathrm{CO}$ & 28 & 2.0 & $14 \%$ \\
\hline $\mathrm{CO}_{2}$ & 44 & 2.92 & $17 \%$ \\
\hline
\end{tabular}
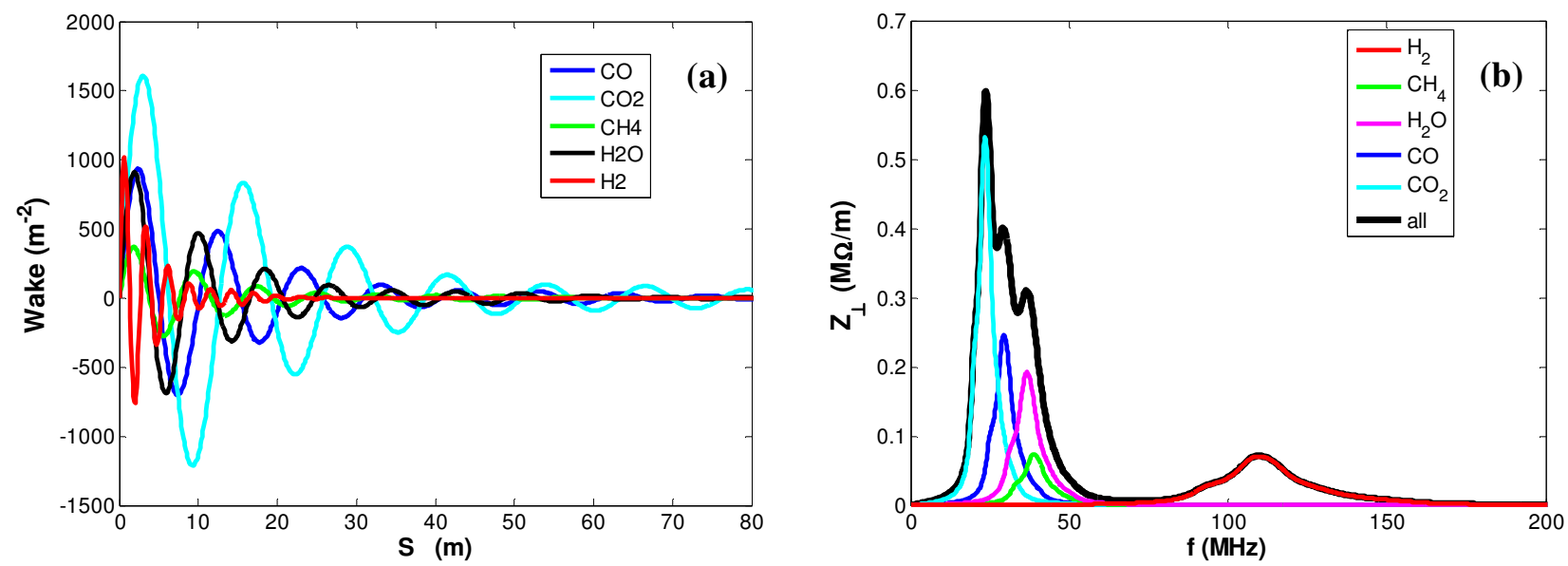

FIG. 1. Wake function (a) and the real part of impedance (b) due to different ion species in SPEAR3 with total pressure of 0.5 nTorr. The vacuum components are listed in Table 2. The beam filling pattern consists of six bunch trains and a total beam current of $500 \mathrm{~mA}$. The total bunch number is 280 .

The ILC beam has three different beam filling patterns as shown in Table 3: so called KCS, DRFS and FP upgrade. The KCS and DRFS have the same number of bunches and beam current. They are different only in the beam filling pattern: KCS has longer bunch train and longer bunch train gap. FP upgrade configuration has the highest beam current and the longest bunch train. Note that the beam filling pattern is not exactly periodic as shown in Table 3 . Besides the filling pattern configuration shown in Table 3, an additional bunch train follows to make the total bunch number as listed. Fig. 2 shows the vertical wake function and impedance of various types of ion species in the KCS case with a total vacuum pressure of 0.5 nTorr. Differently from the SPEAR3 case, the wake function in the ILC damping ring no longer has the resonance form as shown in Eq. (1) although it is a superposition of resonance forms. There are three peaks in the impedance of each type of ion species. Different peaks are the contributions of ions from the different sections along the ring, which can be clearly seen from the frequency distribution along the ring as shown in Fig. 3. The large variation of ion frequencies can explain the shape of the wake function and the impedance. The long standard section contributes to the first peak (low frequency end) in the impedance plot while the short (about 200 meters long) wiggler section (with high frequency) contributes the third peak in the impedance. The wake function is proportional to $\left[\sigma_{y}\left(\sigma_{y}+\sigma_{x}\right)\right]^{-3 / 2}$. Therefore the high frequency (small beam size) section is very important although it is short. 
Apparently, there is a lager variation in beam size along the ILC damping ring when compared with that of the SPEAR3 ring. Therefore, a stronger damping of the instability is expected.

Table 3: Beam filling patterns in the ILC DTC damping ring

\begin{tabular}{|c|c|c|c|}
\hline Parameters & KCS & DRFS & FP upgrade \\
\hline Number of Bunches & 1312 & 1312 & 2625 \\
\hline Beam Current[mA] & 389 & 389 & 779 \\
\hline Bunch Spacing [ $\lambda_{R F}$ ] & 4 & 4 & 2 \\
\hline Beam Filling Period & 19 & 14 & 29 \\
\hline Fill Pattern (1period) & & & 44 \\
\hline Train [bunch number] & 34 & 22 & 31 \\
\hline Gap [in $\lambda_{R F}$ ] & 45 & 33 & 45 \\
\hline Train [bunch number] & 34 & 22 & 31 \\
\hline Gap [in $\lambda_{R F}$ ] & 49 & 33 & \\
\hline Train [bunch number] & & 22 & \\
\hline Gap [in $\lambda_{R F}$ ] & & 33 & \\
\hline & & 23 & \\
\hline & & 33 & \\
\hline
\end{tabular}
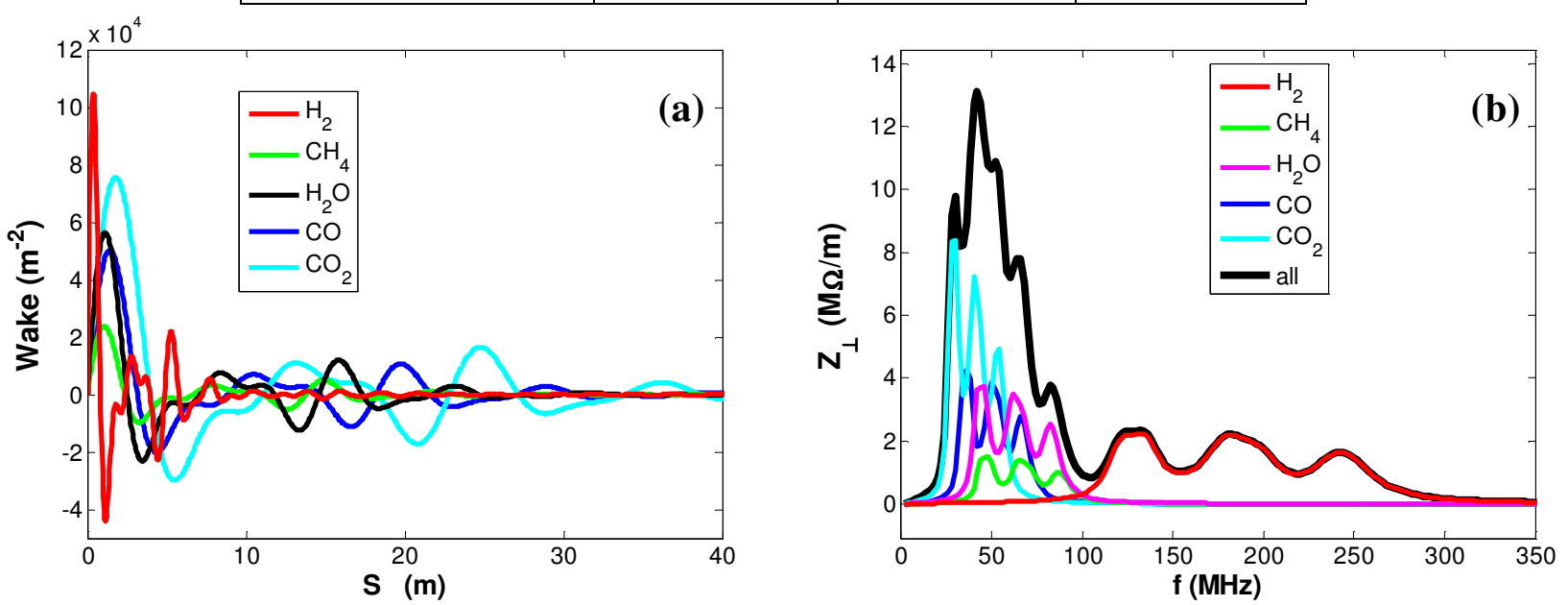

FIG. 2. Wake function (a) and the real part of impedance (b) driven by various ion species in the ILC vacuum chamber with KCS beam. The total vacuum pressure is 0.5 nTorr with partial gas pressures percentage shown in Table 2 .

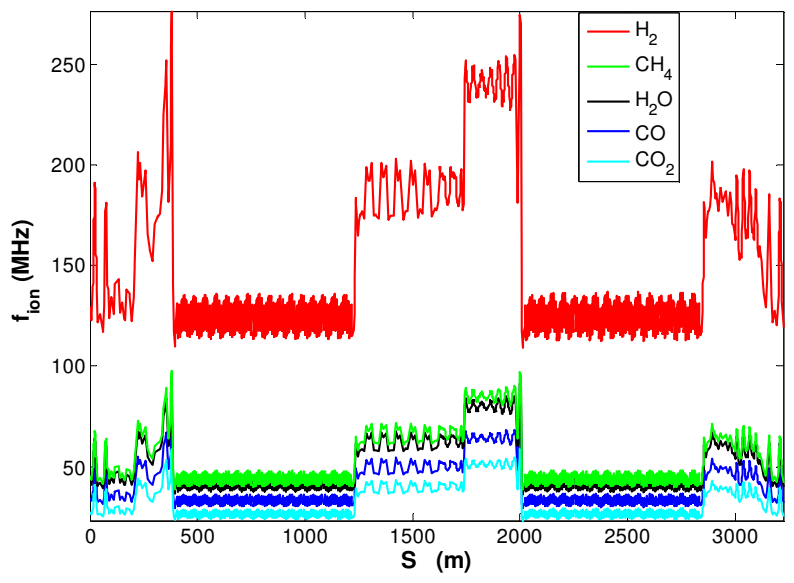

FIG. 3. Calculated vertical ion frequency along the ILC damping ring with the KCS beam. 


\section{ANALYSIS OF INSTABILITY USING ARBITARY WAKE FUNCTION AND IMPEDANCE}

The wake function in the general case with arbitrary beam optics and vacuum is given by Eq.(4). It is straight forward to calculate the instability directly using the wake function. When the beam is evenly filled along the ring, the coherent frequency shift for mode number $\mu$ is [9]

$$
\Omega_{\mu}-\omega_{\beta}=\frac{r_{e} c N_{e}}{4 \pi v_{y} \gamma} \sum_{\mathrm{m}=0}^{\mathrm{M}-1}\left[\sum_{k} W_{y}\left(k C+\frac{m}{M} C\right) e^{2 \pi i v_{\beta} k}\right] e^{2 \pi i\left(\mu+v_{\beta}\right) \frac{m}{M}} .
$$

Here $v_{y}$ and $\omega_{\beta}$ are the betatron tune and frequency, $M$ is the bunch number and $C$ is the circumference, $r_{e}$ is the classical radius of electron and $\gamma$ is the relativistic factor. We first applied the above approach to the study of beam ion instability in the ILC damping ring [10]. Similarly, the coherent frequency shift can also be calculated using the impedance of the ion cloud as

$$
\Omega_{\mu}-\omega_{\beta}=-i \frac{N_{e} M r_{e} c}{2 \gamma T_{0}^{2} \omega_{\beta}} \sum_{p=-\infty}^{\infty} Z_{\perp}\left(\left(p M+v_{y}+\mu\right) \omega_{0}\right)
$$

Here $T_{0}$ and $\omega_{0}$ are the revolution period and frequency. The real part of the frequency shift gives the tune shift due to ion cloud and the imaginary part gives the exponential growth rate of the coupled bunch instability. The growth time of the instability is the inverse of the growth rate. A multi-bunch train beam filling pattern with short train gaps is an effective way to mitigate the beam ion instability. We will use the formulae above to estimate the beam ion instability of multi-bunch train beam with the approximation of a uniformly distributed beam.

\section{Effects of multiple gas species}

Fig. 4 shows the calculated growth rate and tune shift of the unstable modes in SPEAR3 using the wake function and impedance shown in Fig. 1. A negative growth rate means instability. The distribution of the growth rate of the unstable modes can be clearly explained by the impedance. The unstable modes driven by $\mathrm{CO}_{2}{ }^{+}, \mathrm{CO}^{+}, \mathrm{H}_{2} \mathrm{O}^{+}$and $\mathrm{CH}_{4}{ }^{+}$are close to each other, while the unstable modes driven by $\mathrm{H}_{2}{ }^{+}$are well separated. The growth time of the fastest unstable mode is $2.0 \mathrm{~ms}$, which agrees well with the simulated growth time of $1.6 \mathrm{~ms}$. The growth time of the fastest unstable modes driven by $\mathrm{H}_{2}{ }^{+}$is $16 \mathrm{~ms}$, which is longer than the radiation damping time of $5.3 \mathrm{~ms}$. Therefore, the unstable modes driven by $\mathrm{H}_{2}{ }^{+}$are completely suppressed. This agrees with the observation at SPEAR3 where no unstable modes driven by $\mathrm{H}_{2}{ }^{+}$ have been found. The results of Fig. 4 clearly show the reason why superposition rule doesn't apply since the distributions of the unstable modes driven by different ion species are different due to the difference in their oscillation frequencies. The single gas model would overestimate the instability with the simplified assumption that the mode numbers driven by different ion species are the same. Therefore, it is important to use multiple gas species in the 
analysis and simulation. The maximum tune shift $\Delta Q_{y}$ due to the ion cloud is $5 \times 10^{-5}$, which is negligible compared with other impedance effects.

The impedance of ion cloud in the ILC damping ring is widely distributed due to the large variation of the beam size along the ring. This results in a stronger damping to the beam instability. Fig. 5 shows the estimated growth rate and tune shift due to various types of ions with the KCS beam. There is a minimum growth time of $0.89 \mathrm{~ms}$ and maximum tune shift of 0.002 . It agrees with the simulated growth time of $0.61 \mathrm{~ms}$ as shown later. Three unstable regimes appear for $\mathrm{CO}_{2}^{+}, \mathrm{CO}^{+}, \mathrm{H}_{2} \mathrm{O}^{+}$. Each regime of the unstable modes is driven by ions at different sections along the ring as expected from the impedance shown in Fig. $2 \mathrm{~b}$ and the ion frequency distribution in Fig. 3. The frequencies of $\mathrm{CO}_{2}^{+}$, $\mathrm{CO}^{+}, \mathrm{H}_{2} \mathrm{O}^{+}$and $\mathrm{CH}_{4}{ }^{+}$form a continuous spectrum, which ranges from $10 \mathrm{MHz}$ to $100 \mathrm{MHz}$. This wide frequency range contributes to the broadband of unstable modes in Fig. 5. It is interesting to note that the unstable modes around mode 900 driven by $\mathrm{H}_{2}{ }^{+}$are damped, while these modes driven by other ion species are unstable. Thence, the total growth rate including the effect of multiple gas species is actually reduced. Hydrogen ions provide damping to the most unstable modes. Once again, it is crucial to model the beam ion instability using multi-gas species vacuum.

Fig. 6 shows the unstable modes for the FP upgrade beam configuration. The FP upgrade configuration has the shortest growth time of $0.7 \mathrm{~ms}$ due to the highest beam current. The damping effect of $\mathrm{H}^{+}$on the most unstable mode is large in the FP upgrade configuration. The maximum tune shift $\Delta Q_{y}$ due to the ion cloud is 0.0027 . Benefitting from shorter bunch trains, the DRFS configuration (not shown here) has a longer growth time of $1.2 \mathrm{~ms}$ although it has the same beam current as the KCS configuration.

For a multiple bunch train filling pattern, the tune shift induced by the ion cloud varies along the bunch train, which may provides BNS damping to the beam instability [5]. However, this tune spread is very small and its damping effect is negligible in this case.

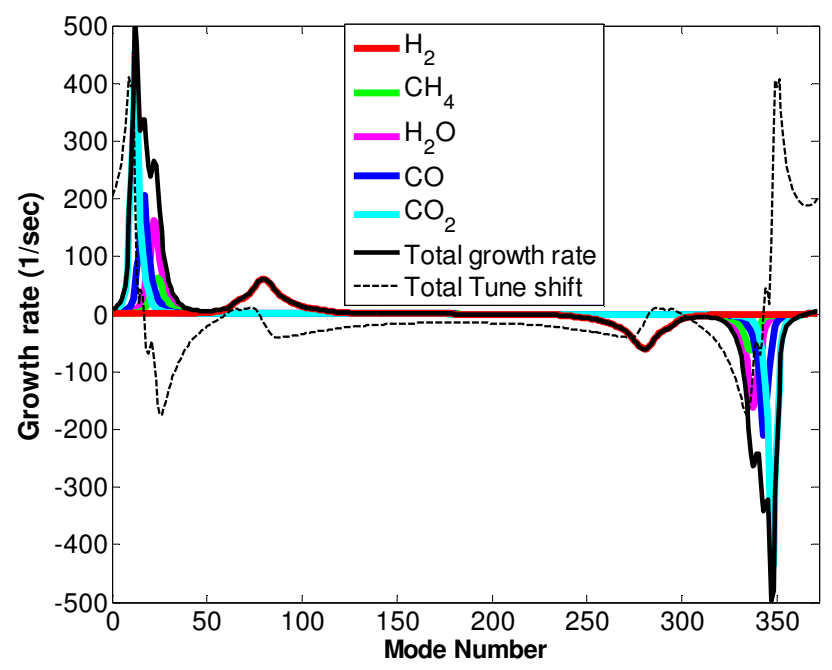


FIG. 4. Growth rate and tune shift in SPEAR3 driven by various ion species as calculated from the wake function and impedance shown in Fig.1. For each gas species, only the growth rates are shown. The growth time of the fastest unstable mode is $2.0 \mathrm{~ms}$. The peak at mode 280 is driven by hydrogen ions, which has a growth time of $16 \mathrm{~ms}$.

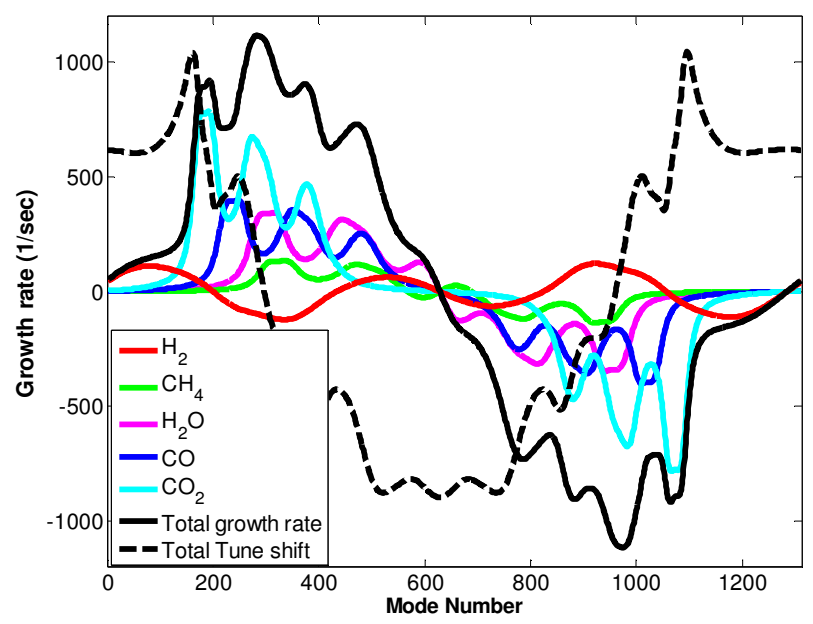

FIG. 5. Growth rate and tune shift due to various ion species for the ILC KCS configuration. For each gas species, only the growth rates are shown. The total vacuum pressure is 0.5 nTorr with partial gas pressures percentages shown in Table 2 .

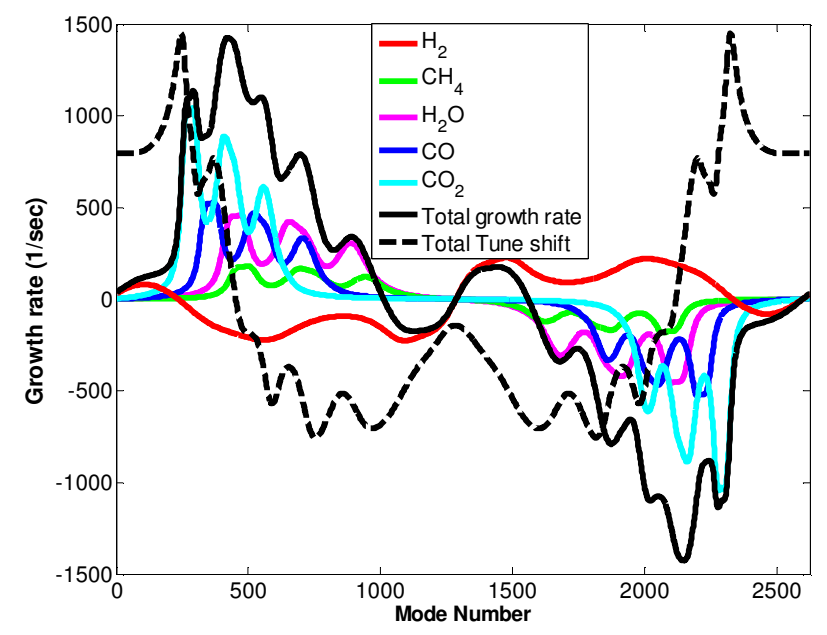

FIG. 6. The unstable modes driven by various types of ions for the ILC FP upgrade configuration. The total vacuum pressure is 0.5 nTorr with partial gas pressures percentages shown in Table 2.

\section{Effects of nonlinear space charge force and beam optics}

Both nonlinear space charge force and the beam size variation due to the beam optics provide damping to the beam ion instability. Therefore, it is interesting to investigate their damping effects independently. The comparison of the effects of nonlinear space charge force and beam optics in SPEAR3 is shown in Fig. 7. Three cases are compared: (1) with nonlinear space charge force only; (2) with beam optics effect only; (3) with both effects. The nonlinear space charge force is turned on and off by a low $Q_{0}$ of 9 and a high $Q_{0}$ of 1000 , respectively. The beam optics effect is 
included or excluded by using variational or constant beam size, respectively. In the case of a constant beam size, an average betatron function is used to calculate the beam size. The figure shows that the nonlinear space charge force and the beam optics effect have a similar damping effect on the beam ion instability in SPEAR3.

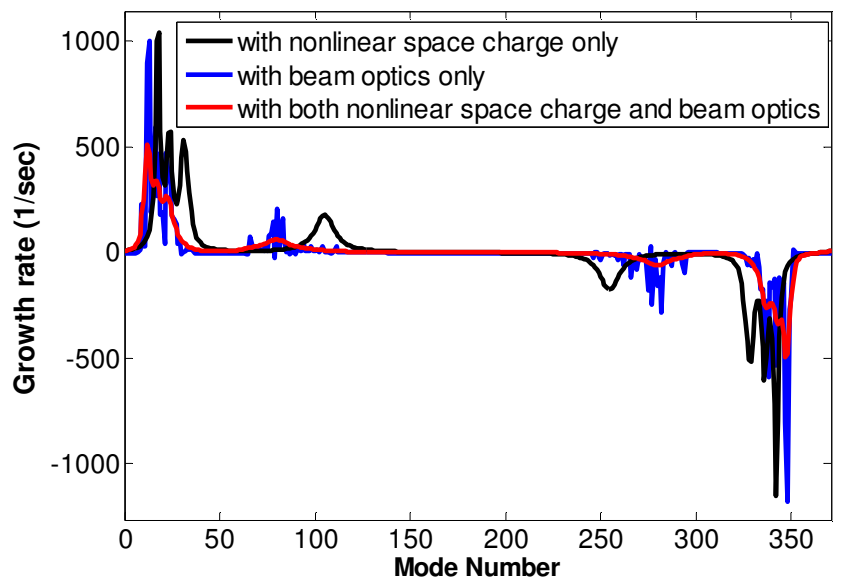

FIG. 7. The growth rate of beam instability in SPEAR3 with different effects: with nonlinear space charge effect only; with beam optics effect only and with both nonlinear space charge force and beam optics effects.

The comparison of the effects of nonlinear space charge force and beam optics in the ILC KCS case is shown in Fig. 8. The large variation in beam size does provide a strong damping to the instability as expected. The damping of the instability due to the variation of beam size is about twice stronger than the nonlinear space charge effect. Generally speaking, both effects are important in the damping of the beam ion instability.
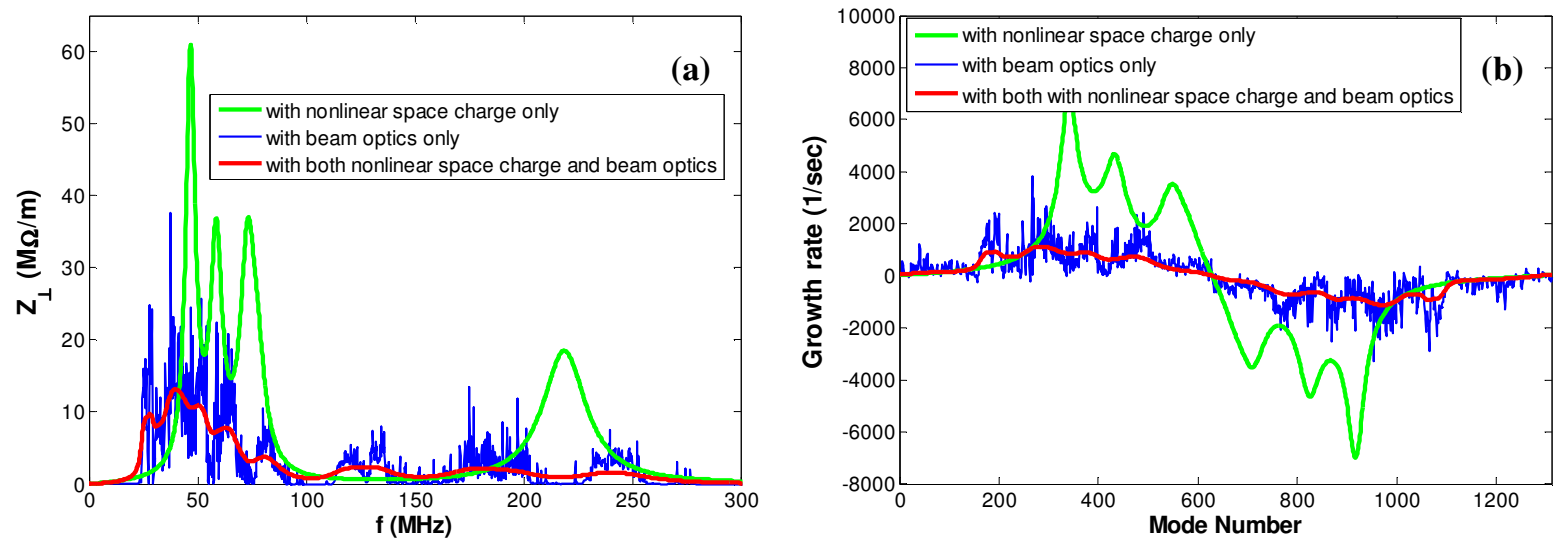

FIG. 8. The real part of the impedance (a) and growth rate of beam ion instability (b) in ILC damping ring with KCS configuration for various effects: with nonlinear space charge effect only; with beam optics effect only and with both nonlinear space charge force and beam optics effects. 


\section{BETTER UNDERSTANDING OF THE UNDERLYING PHYSICS IN FREQUENCY DOMAIN}

Since we have both the wake function and impedance of the ion cloud, the beam instability can be analyzed either in frequency domain using the impedance or in the time domain using the wake function. However, the impedance provides the frequency information which directly relates to the distribution of the unstable modes and the beam optics. As a result, the instability can be more easily explained from the impedance. The impedance provides a deeper understanding of the physics. In this section, we shall study some important characteristics of the beam ion instability in the frequency domain by using the impedance of the ion cloud.

\section{Estimation of the frequency spread due to the beam optics}

It is important to quantify the ion oscillation frequency spread due to the beam optics. It is represented by an $r m s$ relative frequency spread in [3] and $Q$ factor in [7], respectively. However, it is not clear how to accurately calculate them for a given beam optics because the distribution of the ion frequency, as shown in Fig. 9, is far from the standard distribution. For instance, the calculated $r m s$ relative frequency spread in SPEAR is $100 \%$, which is too large (to be used to estimate the beam instability) comparing with the instability study in previous section. The traditional definition of the quality factor $Q$ is

$$
Q=\frac{\omega_{0}}{\Delta \omega_{0}} .
$$

Where $\Delta \omega_{0}$ is the full width at half maximum (FWHM). It is not clear how to define $\Delta \omega_{0}$ for the frequency distribution shown in Fig. 9. One may approximately estimate the $Q$ factor due to beam optics as the ratio of average frequency along the ring $\bar{\omega}$ to the stand derivation $\sigma_{\omega}$

$$
Q_{\text {optics }} \approx \frac{\bar{\omega}}{\sigma_{\omega}} .
$$

For instance, the estimated $Q$ factor of the CO ion shown Fig. 9 is 9.86 for SPEAR3 and 3.97 for the ILC damping ring, respectively. It is a good approximation when comparing with the instability results in Section III and the accurate method in the following. 


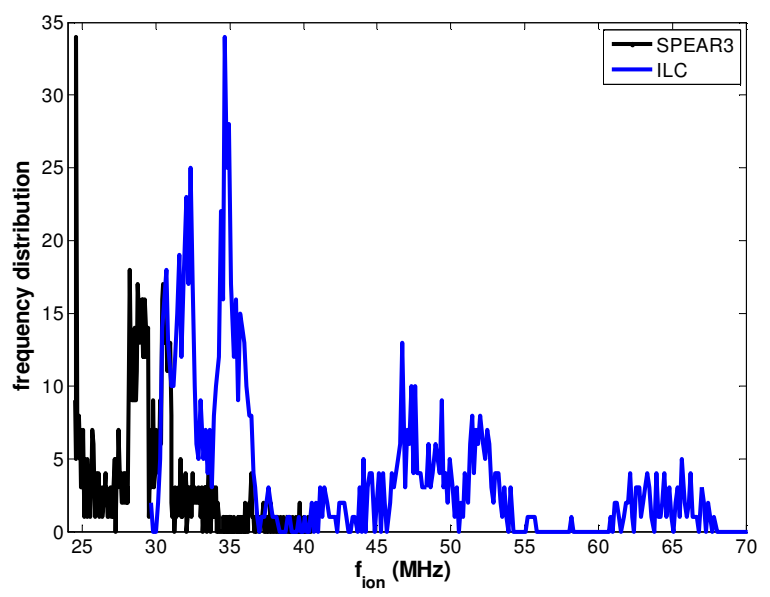

FIG. 9. The distribution of CO ion vertical frequency in SPEAR3 and the ILC damping ring.

Here we provide one method to accurately estimate the $Q$ factor and frequency spread due to the beam optics. For a ring with small variation in beam size, like SPEAR3, the wake function integrated along the whole ring still have the resonance form as shown in Fig.1a. In this case, the wake function due to the ions along the whole ring can be written in the following form

$$
W_{\text {ring }}(s)=W_{0} e^{-\frac{\omega_{r} s}{2 Q_{r i n g} c}} \sin \left(\frac{\omega_{r} s}{c}\right)=\left[W_{0} e^{-\frac{\omega_{r} s}{2 Q_{0} c}}\right] e^{-\frac{\omega_{r} s}{2 Q_{\text {opicis }} c}} \sin \left(\frac{\omega_{r} s}{c}\right)
$$

where $Q_{\text {ring }}$ is the $Q$ factor of the wake function due to the ion cloud along the whole ring and $Q_{\text {optics }}$ is due to the variation of beam size along the ring. We can get the following relationship from Eq.(11)

$$
\frac{1}{Q_{\text {ring }}}=\frac{1}{Q_{0}}+\frac{1}{Q_{\text {optics }}} \text {. }
$$

The $Q$ factor $Q_{\text {ring }}$ can be found by fitting of the wake function along the whole ring. For the wake function of each type of ion species shown in Fig.1a, we get $Q_{\text {ring }}=4.49$ by fitting and $Q_{\text {optics }}=8.9$ from the above equation. Fig. 10 shows the wake function of CO ion in SPEAR3 and the fitting to the resonance form. The wake function along the whole ring does have perfect resonance form. Note that the $Q_{\text {optics }}$ in SPEAR3 is close to $Q_{0}$ of 9.0 due to the nonlinear space charge effect. This finding agrees with the analysis in the previous section where the beam optics and nonlinear space charge force contribute to a similar damping to the instability. The $Q_{\text {optics }}$ represents the relative variation of the ion frequency due to the beam optics effect. It doesn't vary with ion species as expected. As a results, the total wake function (impedance) of ion cloud in SPEAR3 can be simply represented by 5 (due to 5 gas species in the model) resonance-type wake function (impedance). 
It should be pointed out that the wake function due to ions along the whole ring might not be well represented by single resonance mode with the form of Eq. (1) if there is a large frequency variation of the ions along the ring, for instance in the ILC damping ring. In this case, a few resonance-type wake functions with different frequency and $Q$ can be used to represent the total wake function along the whole ring. The total wake function, for instance, can be separated into the wake functions in the arc, wiggler and straight sections. Apparently, the impedance driven by each type of gas species in the ILC damping ring shown in Fig.2 consists of 3 resonance modes. One can estimate the $Q$ values $\left(Q_{\text {ring }}\right.$ and $Q_{\text {optics }}$ ) of each resonance mode following the methods above.

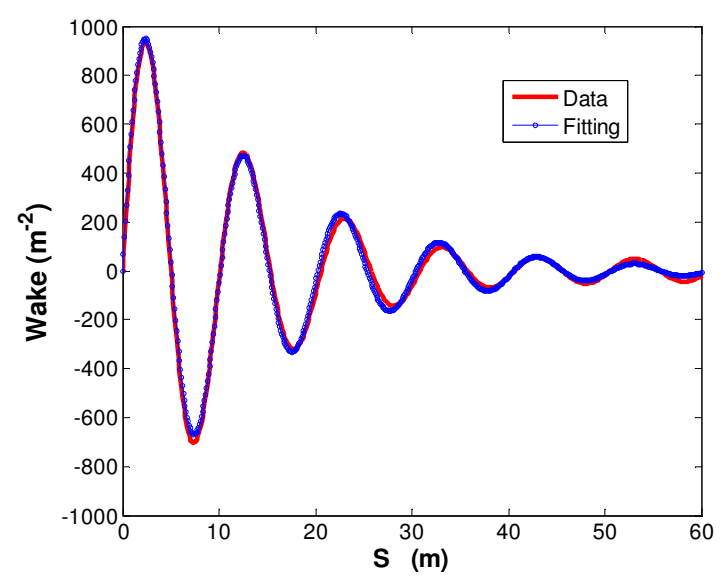

FIG. 10. Wake function of CO ion along the whole SPEAR3 ring for a beam current of 500mA. The wake function is perfertly fitted to a resonant type form with a resonant requency of $29.58 \mathrm{MHz}$ and $Q$ of 4.485 .

\section{The direct relation of the instability growth rate with the impedance}

One may notice that the distribution of unstable modes is similar to the distribution of impedance as shown before. Indeed, they are closely related. Now we look at the detail. For a resonance-type wake function shown in Eq.(1), the Fourier Transform gives the impedance in the frequency domain as

$$
Z_{i o n}(\omega)=\frac{\hat{W}_{t}}{\omega} \kappa \frac{Q}{1+i Q\left(\frac{\omega_{i}}{\omega} \frac{1}{\kappa}-\frac{\omega}{\omega_{i}} \kappa\right)} \approx \frac{\hat{W}_{t}}{\omega} \frac{Q}{1+i Q\left(\frac{\omega_{i}}{\omega}-\frac{\omega}{\omega_{i}}\right)}
$$

Here $\hat{W}_{t}$ and $Q$ are the amplitude and quality factor obtained by fitting the wake function given by Eq.(4) according to

the resonance model in $\operatorname{Eq}(1)$. The factor $\kappa=\sqrt{1-(1 / 2 Q)^{2}} \approx 1$ in most cases. At the resonance, $\omega=\omega_{i}$, the real part of the effective impedance can be simplified to

$$
\operatorname{Re}\left\{\sum_{p=-\infty}^{\infty} Z_{i o n}\left(\left(p M+v_{y}+\mu\right) \omega_{0}\right)\right\}=\operatorname{Re}\left(Z_{i o n}\left(\omega_{i}\right)\right) \approx \frac{\hat{W}_{t}}{\omega_{i}} Q
$$


Substituting the above equation into Eq.(8), we obtain the maximum growth rate of the instability

$$
\frac{1}{\tau} \approx \frac{N_{e} M r_{e} c}{2 \gamma T_{0}^{2} \omega_{\beta}} \operatorname{Re}\left(Z_{\text {ion }}\left(\omega_{i}\right)\right) \approx \frac{N_{e} M r_{e} c}{2 \gamma T_{0}^{2} \omega_{\beta}} \frac{\hat{W}_{t}}{\omega_{i}} Q .
$$

The above equation reveals important physics: the growth rate of an unstable mode is proportional to the impedance at that frequency. This explains why the distribution of the growth rates of the unstable modes is similar to the impedance distribution as shown in previous sections. Therefore as shown in Fig. 1, the impedance can directly tell about the contribution of the unstable modes from different ion species. On the other hand, the wake function could not reveal it. For instance, the $\mathrm{H}_{2}$ ion in SPEAR3 gives a long growth time although its wake function has the second largest amplitude. The growth rate is proportional to $\hat{W}_{t} Q / \omega_{i} \sim \hat{W}_{t} / \Delta \omega_{i}$. The $Q$ factor is the same for all types of ion species. The large frequency of $\mathrm{H}_{2}$ ion and therefore a large frequency spread, which means a fast decay of the wake function, causes a small growth rate of the instability. Therefore Eq.(15) provides an important information that heavy ions are relatively more important to the beam ion instability due to their small frequency and hence a large impedance. Meanwhile heavy ions have large ionization cross-section and the bunch train gaps are less effective at reducing the density of heavy ions. All these factors make heavy ions much more important for the instability. For instance, in SPEAR3 the fastest unstable mode is driven by $\mathrm{CO}_{2}$ ion, although its partial pressure is only $17 \%$ of the total. If the partial pressure of $\mathrm{CO}_{2}$ is reduced from $17 \%$ to $14 \%$ with the partial pressure of $\mathrm{H}_{2} \mathrm{O} 3 \%$ larger, the growth rate of the fastest unstable mode can be reduced by $15 \%$. In short summary, an accurate vacuum model with multiple gas species is essential to model the beam ion instability. A single gas species vacuum model, as used before for most studies, makes it more difficult to represent realistic vacuum effects.

\section{Effects of bunched beam and chromaticity}

The above analyses are done with a single particle model. For a bunched beam, the interaction force depends on the product of the impedance and the bunching factor. The effective impedance of a bunched beam is given by

$$
Z_{\perp}^{e f f}=\sum_{p=-\infty}^{\infty} Z_{\perp}(\omega) e^{-\left(\omega-\omega_{\xi}\right)^{2} \sigma_{z}^{2} / c^{2}} .
$$

Here the chromaticity frequency $\omega_{\xi}=\omega_{0} \xi / \eta$ is the frequency shift due to the chromaticity $\xi=\Delta Q /(\Delta p / p), \eta$ is the slippage factor defined as $\eta=\alpha-1 / \gamma^{2}$ and $\alpha$ is the momentum compaction factor. With zero chromaticity, the effective impedance of a bunched beam is almost the same as that of a single particle beam because of the low frequency of the ion cloud impedance. Therefore the above analyses are also accurate for a bunched beam. However, a non-zero chromaticity induces frequency shift and reduces the effective impedance seen by the beam. The reduction effect depends on both the bunch length and the chromaticity frequency. It is more effective for machines with a low momentum compaction factor and a long bunch length.

In the nominal operation of SPEAR3, a six bunch train beam filling pattern is used to mitigate the beam ion instability. When beam ion instability appears, which often happens at the beginning of a run after a long shut down, a slightly higher vertical chromaticity is used to completely suppress the instability. Fig. 11 shows the observed vertical low sidebands driven by ions at different vertical chromaticity in SPEAR3. The beam has a single bunch train, which 
consists of 280 bunches with a total beam current of $500 m A$.The sidebands are observed above the RF frequency. The frequency in the plot is the difference of the frequency at sideband and the RF frequency. The horizontal chromaticity is 2.0 in all cases. It clearly shows the damping effect due to a large chromaticity. However, the beam life time drops from 10 hours to 4.7 hours when the chromaticity increases from 2.0 to 7.0 . A larger chromaticity can also reduce the beam injection rate. Therefore, the mitigation of beam ion instability with a large chromaticity may be limited in practice due to its side effects.

Fig.12 shows the calculated damping effect of chromaticity on the beam ion instability in SPEAR3. A total vacuum pressure of 0.3 nTorr is used in order to compare with the observation. The instability driven by hydrogen ions is almost negligible even with a small chromaticity if considering the radiation damping shown by the dashed line in the plot. We rarely observed the sidebands driven by Hydrogen ions and they are weak signal on the noisy level even if we saw some. Note that the damping efficiency becomes large with an increasing chromaticity. For instance, the difference between a chromaticity of 8 and 6 is much larger than the difference between a chromaticity of 2 and 0 . The analysis qualitatively agrees with the observation, especially the damping effect with an increasing chromaticity. The difference in the distribution shape of the unstable modes suggests that a larger number of $\mathrm{CO}_{2}$ ions is used in the analysis. This indicates that the partial pressure of $\mathrm{CO}_{2}$ is less than $17 \%$ in average as shown in Table 2.

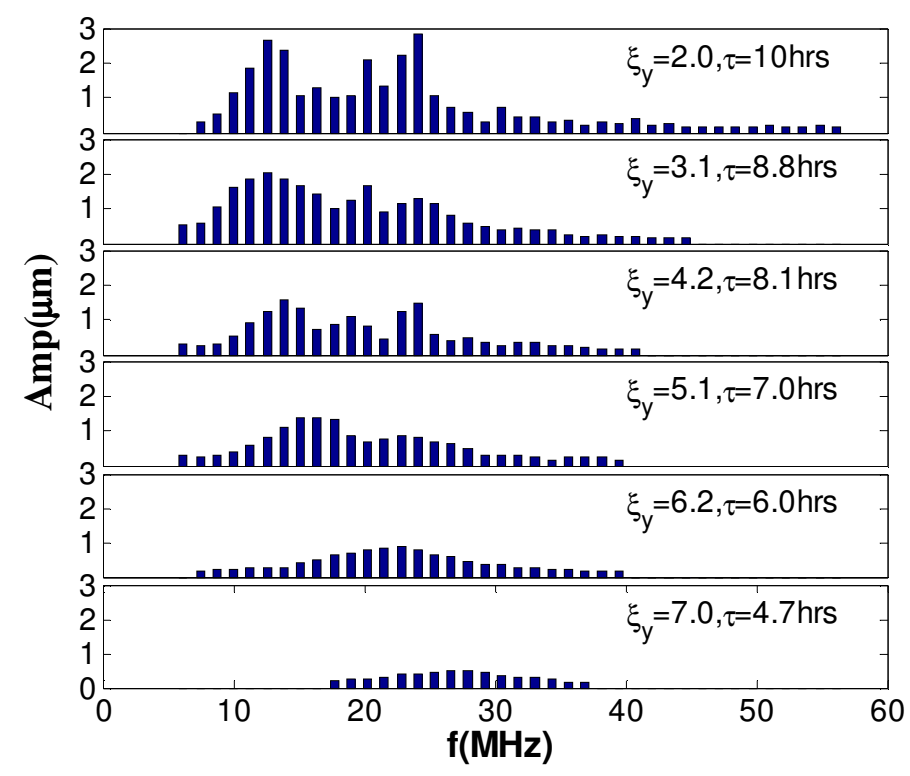

FIG. 11. Observed oscillation amplitude of beam's vertical lower sidebands with varying vertical chromaticity in SPEAR3. The beam has a single bunch train, which consists of 280 bunches with a total beam current of $500 \mathrm{~mA}$ in all the cases. The lifetime is also shown in the plot. 


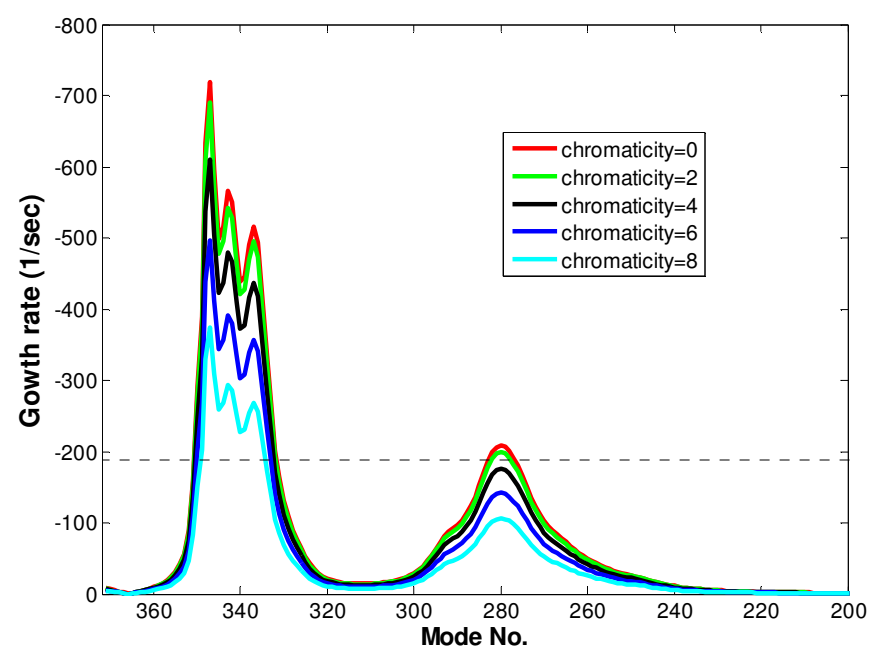

FIG. 12. Calculated growth rate of the beam ion instability in SPEAR3 at different chromaticity. A total vacuum pressure of 0.3 nTorr is used. The beam has a single bunch train, which consists of 280 bunches with a total beam current of $500 \mathrm{~mA}$. The dashed line shows the radiation damping rate.

\section{COMPARISION WITH SIMULATION}

Simulations have a number of advantages in the study of beam-ion instability: the nonlinearity of the ion-cloud force is automatically included; the effects of beam optics and bunch-train gap with arbitrary beam filling pattern can be easily handled; a realistic vacuum model with multi-gas species is straightforward in simulation. A Particle in Cell (PIC) code based on a wake-strong model is used here [7]. The code bas been benchmarked with SPEAR3 experiment [11] and there is a good agreement.

The SPEAR3 vacuum shown in Table 2 with a total pressure of 0.5 nTorr is used in this simulation. A uniform vacuum pressure along the ring is assumed. The simulated beam ion instability in SPEAR3 can be found in [7]. The beam filling pattern consists of six bunch trains with a total beam current of $500 \mathrm{~mA}$. The total vacuum pressure is 0.5 $n$ Torr with the gas species shown in Table 2. The exponential growth time is $1.62 \mathrm{~ms}$, which agrees well with the analysis.

Fig. 13 shows the simulated vertical beam ion instability in the ILC damping ring with KCS configuration. The exact beam filling patterns shown in Table 3, which are not uniform, are used in the simulations. There is an exponential growth when the amplitude of beam oscillation is smaller than the beam size, and then it grows slowly due to the strong nonlinearity of space charge force [12]. The spectrum of unstable modes is broad band and the distribution of the unstable modes agrees well with the analysis shown in Fig. 5. The fastest exponential growth times for the three configurations shown in Table 3 are $0.61 \mathrm{~ms}, 0.91 \mathrm{~ms}$ and $0.40 \mathrm{~ms}$, respectively. Again the simulations agree with our analyses. 

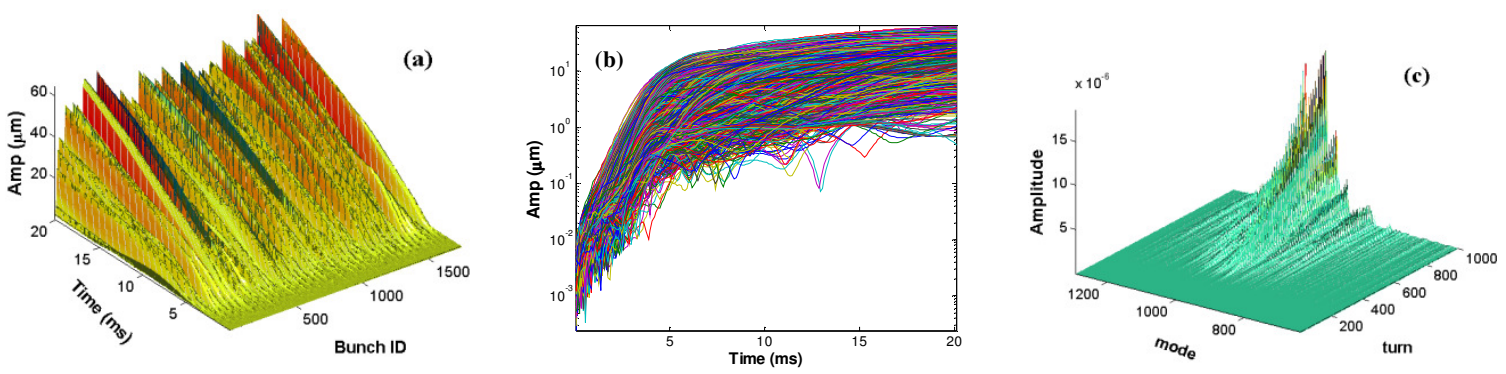

FIG. 13. Simulated vertical beam ion instability in the ILC damping ring with KCS configuration: 3D plot (a) and 2D plot (b) of the growth of vertical amplitude; growth of unstable modes(c). The vertical oscillation amplitude in (a) is in linear scale, while it is in logarithmic scale in (b). The different lines in (a) and (b) are for different bunches. There is a total of 1312 bunches. The fastest growth time is $0.61 \mathrm{~ms}$.

\section{SUMMARY AND CONCLUSION}

We analyze the multi-bunch train beam ion instabilities with arbitrary beam optics, multi-gas species vacuum, nonlinear space charge force and realistic beam filling pattern together. All these factors provide damping to the instability. Our analyses agree well with expensive simulation, which has been benchmarked with the observations in SPEAR3.

Our studies show that it is critical to use multiple gas species. The simple superposition rule of adding the largest growth rate of each type of ions overestimates the instability. For instance, the instability driven by Hydrogen ions in SPEAR3 is completely suppressed by radiation damping. The Hydrogen ions in the ILC damping ring can even provide damping to the most unstable modes.

The damping of the beam ion instability due to beam optics can be clearly seen in the analysis. A beam optics with large variation in beam size, such as the ILC damping ring, SuperB and SuperKEKB, provides stronger damping comparing with beam optics in light sources like SPEAR3. The damping effect can be simply represented by a $Q$ factor. We introduced one accurate method to estimate the $Q$ factor due to the beam optics, which is about 8.9 for the SPEAR3 beam optics.

The nonlinear space charge force also plays an important role in the damping of the beam ion instability; in SPEAR3 it has similar damping effect as the beam optics. In the ILC damping ring, the damping of the instability due to the beam optics is about twice stronger than the nonlinear space charge effect benefiting from the larger variation of the beam size along the ring.

The analyses of beam ion instability have been done in both frequency domain using impedance and time domain using wake function. We introduce the impedance model in the study of beam ion instability for the first time. The impedance of the ion cloud is broadband due to the variation of beam size along the accelerators and the multiple gas species in the vacuum. The impedance provides clear information for understanding the ion instability and the effect of beam optics. One important conclusion is that light ions contribute weakly to the instabilities due to their large oscillation frequency. Other two factors also make light ions less important: the ionization cross-section of light ions is small and the multiple bunch train filling pattern is very effective at reducing the density of light ions. Therefore, the heavy ions are much more important for the beam ion instability. 
A large chromaticity can mitigate the instability at the expense of reduced lifetime. Chromaticity is more effective for machines with a low momentum compaction factor and a long bunch length.

\section{ACKNOWLEDGEMENTS}

We would like to acknowledge the SPEAR3 team for its great help on the SPEAR3 observations. This work is supported by US Department of Energy with Contract No. DE-AC02-76SF00515.

\section{REFERENCES}

[1] T. O. Raubenheimer and F. Zimmermann, Phys. Rev. E52, No. 5, 5487 (1995).

[2] G.V. Stupakov, T.O. Raubenheimer, F. Zimmermann, Phys. Rev. E52, 5499 (1995)

[3] G. V. Stupakov, KEK Proceedings 96-6, 243 (1996)

[4] R. A. Bosch, Physical Review Special Topics - Accelerators and Beams, 3, 034402 (2000)

[5] D. V. Pestrikov, Phys. Rev. ST Accel. Beams 2, 044403(1999).

[6] E.S. Kim and K.Ohmi, Japanese Journal of Applied Physics 48, 086501 (2009)

[7] L. Wang, et al., Physical Review Special Topics - Accelerators and Beams, 14, 084401 (2011)

[8] https://wiki.lepp.cornell.edu/ilc/bin/view/Public/DampingRings/WebHome

[9] A. Chao, "Physics of Collective Beam Instabilities in High Energy Accelerators", Wiley, (1995)

[10]L. Wang and M. Pivi, in the proceedings of 2012 International Particle Accelerator Conference, New Orleans, Louisiana, USA(2012)

[11]L. Wang, et al., p814, Proceedings of IPAC2011, San Sebastián, Spain(2011)

[12] S. Heifets, et. al., SLAC-PUB-12959(2007) 